
\title{
25 Research Soure \\ Lockdowns Lose One Third of Their Impact on Mobility in a Month
}

\section{Yogesh Joshi ( $\nabla$ yjoshi@umd.edu )}

University of Maryland, College Park

\section{Andres Musalem}

University of Chile

\section{Research Article}

Keywords: coronavirus (COVID-19) pandemic, subsequent evolution of mobility, policy makers anticipate

Posted Date: July 20th, 2021

DOl: https://doi.org/10.21203/rs.3.rs-701885/v1

License: (c) (1) This work is licensed under a Creative Commons Attribution 4.0 International License. Read Full License

Version of Record: A version of this preprint was published at Scientific Reports on November 22nd, 2021. See the published version at https://doi.org/10.1038/s41598-021-02133-1. 


\section{Abstract}

As the novel coronavirus (COVID-19) pandemic spread across the world over the past year, many countries imposed lockdowns in the form of stay at home requirements on their citizens to mitigate its spread. We analyze mobility data from 93 countries implementing lockdowns to investigate their effectiveness in immediately reducing mobility and the subsequent evolution of mobility. We find that at the start of a lockdown, median mobility is reduced to $36 \%$ below the baseline, and by another $18 \%$ in the subsequent two weeks. 70 countries had lockdowns lasting beyond four weeks and showed a significant reduction in mobility compared to that prior to the lockdown. Mobility was at its minimum 18 days into the lockdown for the median country. Comparing this minimum mobility to the average mobility two weeks before the lockdown, we observe a median reduction of 50 percentage points, evidencing that lockdowns are effective in reducing mobility. For 59 of these 70 countries, lockdowns lasted at least four weeks after reaching minimum mobility and most observed a significant rebound in mobility during the lockdown period. For the median country, $30.1 \%$ of the mobility reduction achieved is lost within four weeks, and lockdowns lose all their effectiveness in 112.1 days. Overall, our findings show that while lockdowns are effective at significantly reducing mobility, they are also subject to fatigue as the lockdown periods extend longer. The magnitude of lockdown reductions achieved and fatigues reported in this research can help policy makers anticipate the likely longevity of their lockdown policies and help them become more effective by accounting for lockdown fatigue.

\section{Introduction}

The United States recorded its first confirmed case of the novel coronavirus disease (COVID-19) in late January, 2020 in the state of Washington. ${ }^{1}$ By the end of January, thousands of cases were observed in China and cases appeared to be spreading globally, leading the World Health Organization (WHO) to declare COVID-19 to be a Public Health Emergency of International Concern. ${ }^{2}$ By March 2020, COVID-19 had spread to over a hundred countries with over a hundred thousand cases and more than four thousand dead, further leading the WHO to declare it a global "pandemic". ${ }^{3}$

As COVID-19 spread globally, governments in many countries started instituting formal policies with the aim of mitigating the potential outbreak and loss of life within their individual jurisdictions. Starting with travel advisories and travel restrictions, the policies quickly escalated to more severe actions such as stay at home requirements (SHR) which we refer to as lockdowns. The intent of imposing lockdowns was to restrict and reduce the movement of citizens, which in turn would lead to lower contact among people, and hence to lower cases and lesser loss of life. ${ }^{18,19}$ Indeed, scholars have analyzed the impact of mobility on the spread of the disease and shown that a reduction in mobility can slow the spread. $4,5,6,10$

At the same time, many have questioned the appropriateness of governments imposing lockdowns and the general efficacy of lockdowns and quarantines. ${ }^{21}$ The editorial board of the Wall Street Journal publicly opined "These shutdowns are extraordinary and have costs, not least the harm to small business owners. Americans may simply decide to ignore the orders after a time. Absent a more thorough 
explanation of costs and benefits, we doubt these extreme measures will be sustainable for long as the public begins to chafe at the limits and sees the economic consequences". ${ }^{7}$ More generally, research shows that government policy, the alignment of public interests, and compliance interact closely. ${ }^{17,20}$ In this research, we specifically focus our attention on the broader issue of whether and to what extent have lockdowns been effective in terms of achieving their objectives of reducing mobility.

A casual observation of the policies adopted by governments around the world suggests that the severity of the rules as well as the extent of their enforcement has varied significantly across countries. At the same time, the effects that these lockdown policies had on subsequent mobility has also been varied. Motivated by these observations, in this work we investigate the following research questions: [i] How effective are lockdowns in reducing mobility? [ii] Is their effectiveness long-lasting, at least during the time when they are in effect? [iii] If there is variation in their long-term effectiveness across countries, what mobility patterns and country characteristics explain these differences?

Our work contributes to a growing body of research analyzing the impact of government policies on factors such as mobility. ${ }^{12,13}$ Prior research has found that government policies as well as pandemic severity impacts social distancing that is practiced within communities, and that less social distancing is practiced after observing local mitigation. ${ }^{8}$ Researchers have also shown that social distancing and lower population density may be associated with decreased spread of the coronavirus. ${ }^{14}$ Other work has shown that risk attitudes can be a critical factor in predicting mobility reduction, and that regions with more risk averse attitudes may be more likely to change behavior in a pandemic. ${ }^{9}$ Compliance with mobility restrictions have also been shown to be associated with social connections and partisan beliefs. ${ }^{15,16}$ We focus on the impact of lockdowns on mobility and characterize the extent and dynamics of the reduction in mobility achieved by imposing lockdowns in countries across the world.

\section{Empirical Approach}

To study the impact of lockdowns on mobility we analyze policy and mobility data for 116 countries. For each country, we identify the first time that a general requirement to stay at home (i.e., a lockdown) is imposed on its citizens and its duration. Using the metric of mobility at workplaces, we compute a baseline level of mobility prior to the start of the lockdown for that country to understand mobility levels before restrictions went into effect. Using regression models that controls for day-of-the-week effects we characterize the evolution of mobility levels throughout the duration of a lockdown. Via further regression analyses, we assess whether differences across countries in mobility responses to lockdowns can be explained by country characteristics such as demographics and socioeconomic indicators.

\section{Mobility levels are lower during lockdowns}


To understand mobility patterns over the past year, regardless of policy or other interventions, we first compute the median mobility by month in each country for all 116 countries under study and generate a boxplot of these medians by month (Figure 1). During the initial phase, we observe a global median decline in mobility of 10.5 and 47.0 percentage points in March and April 2020 respectively, compared to the baseline reference levels of January-February 2020. This is followed by a pattern of gradual and partial recovery that extends through June 2020 , with mobility levels remaining relatively stable thereafter.

Next, we visualize the implementation of lockdown policies across the world as measured by the proportion of countries in our sample that are under lockdown on any given day (Figure 2). Interestingly, the mobility decline and recovery observed in Figure 1 coincides with the implementation and subsequent relaxation of lockdowns observed in Figure 2. The highest proportion of countries requiring their citizens to stay at home is observed during April 2020 (69.9\%). This proportion stayed at high levels during March to May 2020, and then decreased to lower levels in June 2020, and stayed low for a long time thereafter.

\section{Lockdowns have a strong initial impact and then fatigue sets in}

While at a macro level the prior two figures may suggest that lockdowns are effective, a closer look at the evolution of mobility levels during lockdown periods for each country reveals a more nuanced story. Figure 3 shows that lockdowns achieve a strong initial reduction in mobility but then are followed by a wear out period suggesting that lockdown fatigue is setting in. Across 93 countries that had a lockdown, the median mobility is reduced to $36 \%$ below the baseline at the start of a lockdown, and then by another $18 \%$ two weeks later. Nevertheless, this initial impact exhibits a wear out effect, as mobility gradually rises by approximately 3 percentage points with each additional week that the lockdown stays in effect. After 52 days of lockdown, the median mobility levels rise to $35 \%$ below the baseline, effectively erasing all impact of the lockdown, even though the lockdown continues to stay in effect.

As one may expect, the duration for which lockdowns are imposed varies across countries. Hence, it could be hypothesized that the fatigue observed in Figure 3 (black dots) might arise due to a selection effect if countries for which a lockdown is less effective at reducing mobility lift the lockdown earlier. However, an analysis focusing only on those 50 countries where the lockdown was in place for at least 60 days (red triangles in Figure 3 ) reveals a similar pattern of fatigue.

\section{Lockdowns significantly reduce mobility}

To better understand the dynamic effects of imposing a lockdown on mobility at the country level, we focus on 79 countries where lockdowns lasted at least four weeks. Out of these 79 countries, 70 exhibit a significant mobility reduction $(p<0.01)$ in the first two weeks of the lockdown compared to the two weeks before the lockdown. The median value of this reduction is 40 percentage points. The data exhibits 
strong day of the week seasonality, hence we compute a seven day moving average of mobility level during the lockdown to better understand lockdown dynamics. Based on this metric, the minimum level of mobility is achieved 18.0 days into the lockdown for the median country. Comparing this minimum mobility to the average mobility two weeks before the lockdown, we observe a median reduction of 50 percentage points. These observations indicate that lockdowns are effective in reducing mobility.

\section{$30 \%$ of the effectiveness of a lockdown is lost in four weeks}

For 59 of the 70 countries mentioned above, lockdowns were extended for at least four weeks after reaching the minimum mobility level. For the median country, we find that $30.1 \%$ of the mobility reduction achieved from imposing a lockdown is lost within four weeks after reaching this minimum level. We then specify regression models to explain the observed daily mobility level within a country as a function of the days since the lockdown was imposed, after controlling for day of the week effects (additional details provided under Materials and Methods). For $96 \%$ of these countries, we observe a significant rebound in mobility during the lockdown period. For these countries, we used the fitted models to estimate the number of days it would take a lockdown to reach the average mobility levels observed during the two weeks before the start of the lockdown. This calculation reveals that lockdowns would lose all their effectiveness in 112.1 days for the median country.

\section{Greater mobility reductions are associated with greater lockdown fatigue}

There are important differences across countries in terms of the magnitude of the fatigue they exhibit. This is shown in Figure 4, which displays for each country the average mobility level one week before the start of the lockdown and four weeks after reaching the minimum mobility level during the lockdown. We obtain a marginally significantly negative correlation between both metrics (correlation $=-0.23, p$-value $=$ 0.08 ), which suggests that countries that achieved lower mobility levels prior to the start of a lockdown exhibited a greater rebound in mobility.

Similarly, Figure 5 replaces the horizontal axis in Figure 4 by the reduction in mobility achieved between the start of a lockdown and the date when the minimum mobility level is reached during the lockdown. We obtain a significantly positive correlation between both metrics (correlation $=0.25, p$-value $=0.05$ ), which suggests that countries that achieved a greater reduction in mobility from the start of a lockdown exhibited a greater rebound in mobility.

\section{Country characteristics and mobility dynamics}

We observe substantial variation in mobility dynamics during the lockdown across countries. We next explore whether country characteristics help explain some of this variation. We focus on three mobility 
metrics: i) mobility levels observed prior to the lockdown; ii) the reduction in mobility achieved between the two weeks prior to the lockdown and the date when the minimum mobility is achieved; and iii) the rebound in the mobility levels observed four weeks after reaching the minimum mobility levels during the lockdown. We conduct regression analyses with each of these three metrics as a dependent variable and country characteristics as predictors (please refer to the methods and materials section for a list of the country characteristics used for each regression).

First, in terms of the analysis of mobility levels prior to the lockdown, we find that both the policies adopted by a country before a lockdown as well as its education levels are significant predictors for this metric. In particular, we obtain a significantly negative impact of the stringency index observed prior to the lockdown and the mean years of schooling of a country.

Second, considering the reduction of mobility achieved between the start of a lockdown until reaching the minimum mobility level, once again the policies implemented prior to the start of the lockdown significantly predict this mobility reduction. More specifically, more stringent policies prior to the lockdown are associated with a weaker drop in mobility. In addition, countries with populations enjoying a greater life expectancy are associated with stronger reductions in mobility.

Third, regarding the mobility rebound realized four weeks after reaching the minimum level, both the mobility levels observed prior to the start of the lockdown and the mobility reduction achieved during lockdown are significant predictors with opposite relationships. On the one hand, greater mobility levels prior to the start of a lockdown are associated with a weaker mobility rebound. On the other hand, a greater mobility reduction achieved during the lockdown is associated with a stronger mobility rebound.

\section{Discussion}

Overall, our global analysis suggests that lockdowns work, in that imposing a lockdown is associated with a significant reduction in the observed levels of mobility for most countries in our analysis. Interestingly though, mobility dynamics in response to a lockdown vary significantly across the world. We observe and quantify a global pattern of lockdown fatigue, as mobility levels slowly start rising the longer a lockdown stays in effect. In a relatively short of amount of time (e.g., four weeks), lockdown fatigue may eliminate one third of the gains achieved in terms of reduced mobility. A direct implication of this result may be that lockdown compliance might need to be reinforced by governments when lockdowns remain in effect for long periods of time.

In terms of the drivers of lockdown fatigue, the strongest predictor appears to be the reduction in mobility achieved during the lockdown: the greater this reduction, the stronger the fatigue. Accordingly, these two appear to be compensatory. A similar association is observed between lockdown fatigue and the mobility levels prior to the start of the lockdown. Countries that had already achieved lower levels of mobility prior to a lockdown exhibit greater mobility rebounds during their lockdown periods. Interestingly, lower 
mobility levels prior to a lockdown are observed for countries with greater education levels. However, these countries are likely to experience greater lockdown fatigue.

The effectiveness of lockdowns is also associated with other country characteristics such as the life expectancy enjoyed by the population in each country. Countries with greater life expectancy show stronger mobility reductions during a lockdown. The opposite is observed for countries that had more strict policies prior to the implementation of a lockdown.

Our analysis has several limitations. First, we do not intend to make any causal claims; our findings are based on summarizing and regressing available data across sources to study the interplay between government policy and mobility. Second, our inferences of mobility are based on workplace mobility data, and while other mobility types have a strong positive correlation with this metric, it may be beneficial to further investigate the impact of lockdowns on other types of mobility. Finally, in this research we do not incorporate the explicit impact of case data on mobility. It is likely that beyond policy, observing actual cases in their communities may have a further impact on people's decisions to move around. Nevertheless, we hope that our findings above help inform policy makers on the nature of response they may expect when implementing lockdowns and incorporate these observations for more effective planning purposes.

\section{Materials And Methods}

Data: The data used in this research was compiled from three sources:

[1] Google COVID-19 Community Mobility Reports: This dataset was accessed on March $2^{\text {nd }} 2021$, and provides daily mobility data, relative to a baseline level of mobility as measured during a reference period of January 3 to February 6, 2020. Mobility measures were constructed by Google using mobile devices of users who have opted in to Location History for their Google accounts. There are six distinct mobility measures: workplace, retail \& recreation, grocery \& pharmacy, parks, transit stations and residential. The first five are highly and positively correlated with each other, while the sixth is negatively correlated with the first five measures. Of these measures, workplace mobility exhibits the fewest missing values, hence we use this measure as our metric for mobility. This metric was available starting February 15, 2020 through February 26, 2021.

We focus on data aggregated at the country level, giving us 246 distinct entities labeled as countries, although some of these are territories such as Puerto Rico. For ease of exposition, we refer to these 246 territories as countries. Of these 246 , we exclude countries that have missing values for workplace mobility, leaving us with 116 countries.

For each country, the data also includes characteristics such as population and geographical area.

[2] Oxford COVID-19 Government Response Tracker: ${ }^{11}$ This dataset was accessed on March $2^{\text {nd }} 2021$, and provided daily policy data on when each country (or state in the case of US) began requiring its 
citizens to stay at home. Government policy data regarding stay at home requirements (SHR) is provided on an ordinal scale, where 0 is no measures are in place, 1 is government recommending not leaving the house, 2 is the government requiring not to leave the house with exceptions for daily exercise, grocery shopping, and 'essential' trips; and 3 is the government requiring not leaving house with minimal exceptions (e.g., allowed to leave once a week, or only one person can leave at a time, etc). We classify SHR levels 2 and 3 as being under a lockdown, since both these levels require a citizen to stay at home. A second variable in this dataset indicates whether SHRs are targeted to specific geographic regions (e.g., certain counties or regions), as opposed to being applied to the general population across the country. The final variable of interest from this dataset is the Stringency Index, which provides a composite score in the range of 0-100 (higher being more stringent) based on nine distinct policies related to school and workplace closings, cancellation of public events and public transport, restrictions on gatherings and on internal movements, on international travel, and whether the government is running public information campaigns.

[3] The United Nations Development Project (UNDP) data: This dataset was accessed on March $9^{\text {th }}, 2021$, and contains country level data on gross national income per capita, life expectancy at birth in years, expected years of schooling, mean years of schooling. When merging this data set with the previous ones, we manually checked for matching country names where needed.

\section{Methods}

The analysis for each country begins by identifying the first date when a lockdown is applied to the general population. Hence, we find the earliest date for which the variable C6_Stay at home requirements is at a level of 2 or 3 and the C6_Flag variable equals 1 . The end and hence the duration of this first stayat-home requirement is determined by finding the first date after the beginning of the requirement for which either C6_Stay at home requirements is at a level of 0 or 1 or the C6_Flag equals 0 . Let the $s_{i}$ and $e_{i}$ denote the start and end of the lockdown for country $i$. Both variables are measured from the beginning of the 2020 calendar year. For example, a lockdown starting on March $15^{\text {th }} 2020$ and ending on March $29^{\text {th }} 2020$ would yield values of $s_{i}$ and $e_{i}$ equal to 74 and 88 , respectively. We consider all periods $t$ between the beginning and end of the lockdown for each country and we define the following six dummy variables Monday $_{t}, \ldots$, Saturday $_{t}$, which are equal to 1 if the period corresponds to a Monday,..., Saturday, respectively, and otherwise these variables are equal to 0 . With these definitions, for each country $i$ with a lockdown period of at least 3 weeks we first estimate the following linear model of workplace mobility $\mathrm{m}_{\text {it }}$ for country $i$ in period $t$.

$\mathrm{m}_{i t}=\mathrm{a}_{\mathrm{i}}+\beta_{\mathrm{i}}\left(t-s_{i}\right)+\pi_{1}$ Monday $_{t}+\pi_{2}$ Tuesday $_{t}+\pi_{3}$ Wednesday $_{t}+\pi_{4}$ Thursday $_{t}+\pi_{5}$ Friday $_{t}+\pi_{6}$ Saturday $t_{t}+\mathrm{e}_{i t}$ where $t$ in $s_{i, . .,} e_{i}$

In this linear model, $a_{i}$ measures the expected mobility at the beginning of the lockdown, while $\beta_{i}$ measures the change in mobility as the lockdown for country $i$ is extended for an additional day. Hence, 
the latter coefficient yields a measure of fatigue in terms of the daily wear out effect of the lockdown for country i. Finally, $\mathrm{e}_{i t}$ represents the error term of the model for country $i$ in period $t$.

We then estimate a more flexible version of this model allowing for a change in the trend slope where:

$\beta_{i}[t]=\beta_{i 0}+\beta_{i 1} \mid\left\{t-s_{i}>c_{i}\right\}$,

where $I\{\}$ is an indicator function equal to 1 if the time between the start of the lockdown and period $t$ is greater than the changepoint $c_{i}$. We use the segmented package in $\mathrm{R}$ to estimate the value of both of the slope parameters $\left(\beta_{i 0}\right.$ and $\left.\beta_{i 1}\right)$ and the change point $\left(c_{i}\right)$ for each country $i$. For every country we compare the Akaike Information Criterion (AIC) of this model against a restricted model without a changepoint (i.e., $\left.\beta_{i 1}=0\right)$, selecting the specification with the smallest AIC for that country.

The selected model is used to assess whether each country exhibited a significant rebound in workplace mobility. This is achieved by verifying whether $\beta_{i 0}$ or $\beta_{i 0}+\beta_{i 1}$ are significantly positive. The selected model is also used to estimate the number of days after reaching the minimum mobility during the lockdown $\left(\right.$ minwm $\left._{i}\right)$ it would take to return to the average mobility levels observed in the two weeks prior to the start of the lockdown (avgwm2wprior ${ }_{i}$ ). This is calculated for countries with significant mobility rebounds as follows:

1. When the best fitting model does not have a changepoint and $\beta_{i 0}$ is significantly positive: (avgwm 2 wprior $_{i}-$ minwm $\left._{i}\right) / \beta_{i 0}$.

2. When the best fitting model has a changepoint, $\beta_{i 0}$ is significantly positive, $\beta_{i 0}+\beta_{i 1}$ is not significantly positive: (avgwm 2 wprior $\left._{i}-\operatorname{minwm}_{i}\right) / \beta_{i 0}$. In this case, we must verify whether this value is smaller than the country's changepoint $\mathrm{c}_{i}$. If it is not smaller, then the estimated rebound does not reach the mobility levels prior to the lockdown and hence we don't consider this value.

3. When $\beta_{i 0}$ is not significantly positive, but $\beta_{i 0}+\beta_{i 1}$ is significantly positive: $c_{i}+\left({\text { avgwm } 2 \text { wprior }_{i}{ }^{-}}^{-}\right.$ $\left.\operatorname{minwm}_{i}\right) /\left(\beta_{i 0}+\beta_{i 1}\right)$

4. When both $\beta_{i 0}$ and $\beta_{i 0}+\beta_{i 1}$ are significantly positive: If (avgwm2wprior ${ }_{i}-$ minwm $\left._{i}\right) / \beta_{i 0}>c_{i j}$ then the days to return to the baseline level are calculated as

$\mathrm{c}_{i}+\left(\right.$ avgwm2 wprior $_{i}-$ estwmobc $\left._{i}\right) /\left(\beta_{i 0}+\beta_{i 7}\right)$, otherwise this value is equal to (avgwm2 wprior $_{i}-$ minwm $\left._{i}\right) /$ $\beta_{i 0}$, where estwmobc $c_{i}$ is the predicted workplace mobility for country $i$ at the regression changepoint $c_{i}$.

We use country characteristics to explain differences in the mobility at the beginning of the lockdown, the fatigue or wear out effect and the mobility drop observed at the beginning of the lockdown. We rely on the following country characteristics, with their respective sources in parenthesis:

1. wm7before: 7-day moving average of the workplace mobility evaluated $\mathbf{7}$ days before the start of the lockdown (Google COVID-19 Community Mobility Reports). 
2. si7before: 7-day moving average of the the stringency index evaluated $\mathbf{7}$ days before the start of the lockdown (Oxford COVID-19 Government Response Tracker).

3. population: country population (Google COVID-19 Community Mobility Reports)

4. area: geographical area (squared kilometers, Google COVID-19 Community Mobility Reports).

5. lifeexp: life expectancy (years, UNDP)

6. gnipc: gross national income per capita (UNDP)

7. expsch: expected years of schooling (UNDP)

8. meansch: mean years of schooling (UNDP)

In order to select the explanatory variables to use in each model, we rely on a stepwise regression with both addition and removal of explanatory variables in each step according to a p-value of 0.3 for entry and removal. We obtain identical conclusions if we use instead a p-value of 0.1 for inclusion and removal. The estimation is implemented in the $\mathrm{R}$ statistical computing and graphics software using the ols_step_both_p function. Detailed results are presented below.

The first model we estimate explains differences across countries in their mobility levels prior to the lockdown start, as measured by wm7before. Results are shown in Table 1.

The second model considers the reduction in mobility achieved during the lockdown as measured by the difference between avgwm 2 wprior $_{i}$ and minwm $_{i}$ which we denote as: mobdropbefmin ${ }_{i}$. Table 2 shows the estimated model.

The third model considers the rebound in mobility as measured by the difference between the 7-day moving average workplace mobility observed four weeks after reaching the minimum mobility level and minwm $_{i}$. We denote this measure by wmrebound4weeks. We also include the reduction in mobility achieved between the start of the lockdown and the date when the minimum mobility is achieved (wmdropstartmin) as a regressor in this model. Table 3 shows the estimation results this model.

\section{Declarations}

\section{Author Contributions}

Both authors contributed equally to this research.

\section{Acknowledgements}

Andres Musalem acknowledges partial funding from ANID AFB180003.

\section{Competing Interests}


The authors declare no competing interests.

\section{Data Availability}

The data that support the findings of this study are publicly available online at:

- Google: https://github.com/GoogleCloudPlatform/covid-19-open-data

- Oxford: https://github.com/OxCGRT/covid-policy-tracker

- UNDP: http://hdr.undp.org/sites/default/files/2020_statistical_annex_all.xIsx

\section{Code Availability}

Software used includes R (version 4.0.3) and RStudio (version 1.4.1103) for data analysis and model estimation. The code will be available from the authors upon request after publication (and for review).

\section{References}

1. Rabin, R. C. First Patient With Wuhan Coronavirus Is Identified in the U.S. The New York Times (21 January 2020). https://www.nytimes.com/2020/01/21/health/cdc-coronavirus.html [Accessed 18 March 2021]

2. WHO. Statement on the second meeting of the International Health Regulations (2005) Emergency Committee regarding the outbreak of novel coronavirus (2019-nCoV). The World Health Organization (30 January 2020). https://www.who.int/news/item/30-01-2020-statement-on-the-second-meeting-of-theinternational-health-regulations-(2005)-emergency-committee-regarding-the-outbreak-of-novelcoronavirus-(2019-ncov) [Accessed 18 March 2021].

3. WHO. WHO Director-General's opening remarks at the media briefing on COVID-19. The World Health Organization (11 March 2020). https://www.who.int/director-general/speeches/detail/who-directorgeneral-s-opening-remarks-at-the-media-briefing-on-covid-19--11-march-2020 [Accessed 18 March 2021].

4. Chernozhukov, V., H. Kasahara \& P. Schrimpf. Causal impact of masks, policies, behavior on early covid-19 pandemic in the U.S. Journal of Econometrics 220(1), 23-62 (2021).

5. Wibbens, P. D., W. W-Y. Koo \& A. M. McGahan. Which COVID policies are most effective? A Bayesian analysis of COVID-19 by jurisdiction. PLOS ONE 15(12), e0244177 (2020).

6. Chang, S., Pierson, E., Koh, P.W. et al. Mobility network models of COVID-19 explain inequities and inform reopening. Nature 589, 82-87 (2021).

7. The Editorial Board. The Extreme State Lockdowns. The Wall Street Journal (20 March 2020). https://www.wsj.com/articles/the-extreme-state-lockdowns-11584745130 [Accessed 18 March 2021]. 
8. Pan, Y., Darzi, A., Kabiri, A. et al. Quantifying human mobility behaviour changes during the COVID-19 outbreak in the United States. Sci Rep 10, 20742 (2020).

9. Chan, H.F., Skali, A., Savage, D.A. et al. Risk attitudes and human mobility during the COVID-19 pandemic. Sci Rep 10, 19931 (2020).

10. Brauner, J. M., et al. Inferring the effectiveness of government interventions against COVID-19. Science 371, eabd9338 (2021).

11. Hale, T., Angrist, N., Goldszmidt, R. et al. A global panel database of pandemic policies (Oxford COVID19 Government Response Tracker). Nat Hum Behav (2021). https://doi.org/10.1038/s41562-021-01079-8

12. Xiong C, Hu S, Yang M, Younes H, Luo W, Ghader S, Zhang L. Mobile device location data reveal human mobility response to state-level stay-at-home orders during the COVID-19 pandemic in the USA. J. R. Soc. Interface 17: 20200344 (2020). https://doi.org/10.1098/rsif.2020.0344

13. López, L., Rodó, X. The end of social confinement and COVID-19 re-emergence risk. Nat Hum Behav 4, 746-755 (2020).

https://doi.org/10.1038/s41562-020-0908-8

14. Rubin D, Huang J, Fisher BT, et al. Association of Social Distancing, Population Density, and Temperature With the Instantaneous Reproduction Number of SARS-CoV-2 in Counties Across the United States. JAMA Netw Open. 3(7): e2016099 (2020). doi:10.1001/jamanetworkopen.2020.16099

15. Charoenwong, B., Kwan, A., \& Pursiainen, V. Social connections with COVID-19-affected areas increase compliance with mobility restrictions. Science advances, 6(47), eabc3054 (2020).

16. Clinton, J., Cohen, J., Lapinski, J., \& Trussler, M. Partisan pandemic: How partisanship and public health concerns affect individuals' social mobility during COVID-19. Science advances, 7(2), eabd7204 (2021).

17. Bavel, J.J.V., Baicker, K., Boggio, P.S. et al. Using social and behavioural science to support COVID-19 pandemic response. Nat Hum Behav 4, 460-471 (2020). https://doi.org/10.1038/s41562-020-0884-z

18. Jacobsen GD, Jacobsen KH. Statewide COVID-19 Stay-at-Home Orders and Population Mobility in the United States. World Med Health Policy (2020).

doi: 10.1002/wmh3.350.

19. Jia, J.S., Lu, X., Yuan, Y. et al. Population flow drives spatio-temporal distribution of COVID-19 in China. Nature 582, 389-394 (2020).

https://doi.org/10.1038/s41586-020-2284-y 
20. Lai, S., Ruktanonchai, N.W., Zhou, L. et al. Effect of non-pharmaceutical interventions to contain COVID-19 in China. Nature 585, 410-413 (2020).

https://doi.org/10.1038/s41586-020-2293-x

21. Brooks, S.K., Webster, R.K., Smith, L.E., Woodland, L., Wessely, S., Greenberg, N. and Rubin, G.J. The psychological impact of quarantine and how to reduce it: rapid review of the evidence. The Lancet, 395(10227), 912-920 (2020).

\section{Tables}

Table 1: Estimation results for a linear model of mobility at the start of the lockdown (wm7before) as a function of country characteristics.

\begin{tabular}{llll}
\hline Dependent Variable & wm7before & & \\
\hline Predictor & estimate & t statistic & $\mathrm{p}$-value \\
\hline intercept & 17.99805 & 4.515 & $<0.001$ \\
\hline sibefore & -0.50344 & -8.980 & $<0.001$ \\
\hline meansch & -0.87645 & -2.378 & 0.020 \\
\hline
\end{tabular}

$R^{2}: 0.580$, Adjusted R2: 0.567; F-statistic: 44.85 on 2 and 65 degrees of freedom $(n=68)$.

Table 2: Estimation results for a linear model of lockdown the mobility reduction achieved during a lockdown as a function of country characteristics.

\begin{tabular}{llll}
\hline Dependent Variable & mobdropbefmin & & \\
\hline Predictor & estimate & t statistic & p-value \\
\hline intercept & 34.438 & 1.746 & 0.086 \\
\hline avgsi7bfl & -0.523 & -5.430 & $<0.001$ \\
\hline lifeexp & 0.474 & 1.818 & 0.074 \\
\hline
\end{tabular}

$R^{2}$ : 0.334, Adjusted R2: 0.313; F-statistic: 16.3 on 2 and 65 degrees of freedom $(n=68)$. 
Table 3: Estimation results for a linear model of the mobility rebound as a function of country characteristics.

\begin{tabular}{llll} 
Dependent Variable & \multicolumn{2}{l}{ wmrebound4weeks $_{i}$} & \\
\hline Predictor & estimate & t statistic & p-value \\
intercept & 12.308 & 5.949 & $<0.001$ \\
\hline wmdropstartmin & 0.417 & 2.505 & 0.015 \\
wm7before & -0.220 & -2.353 & 0.022
\end{tabular}

$R^{2}: 0.148$, Adjusted R $R^{2}: 0.118$; F-statistic: 4.86 on 2 and 56 degrees of freedom $(n=59)$.

\section{Figures}

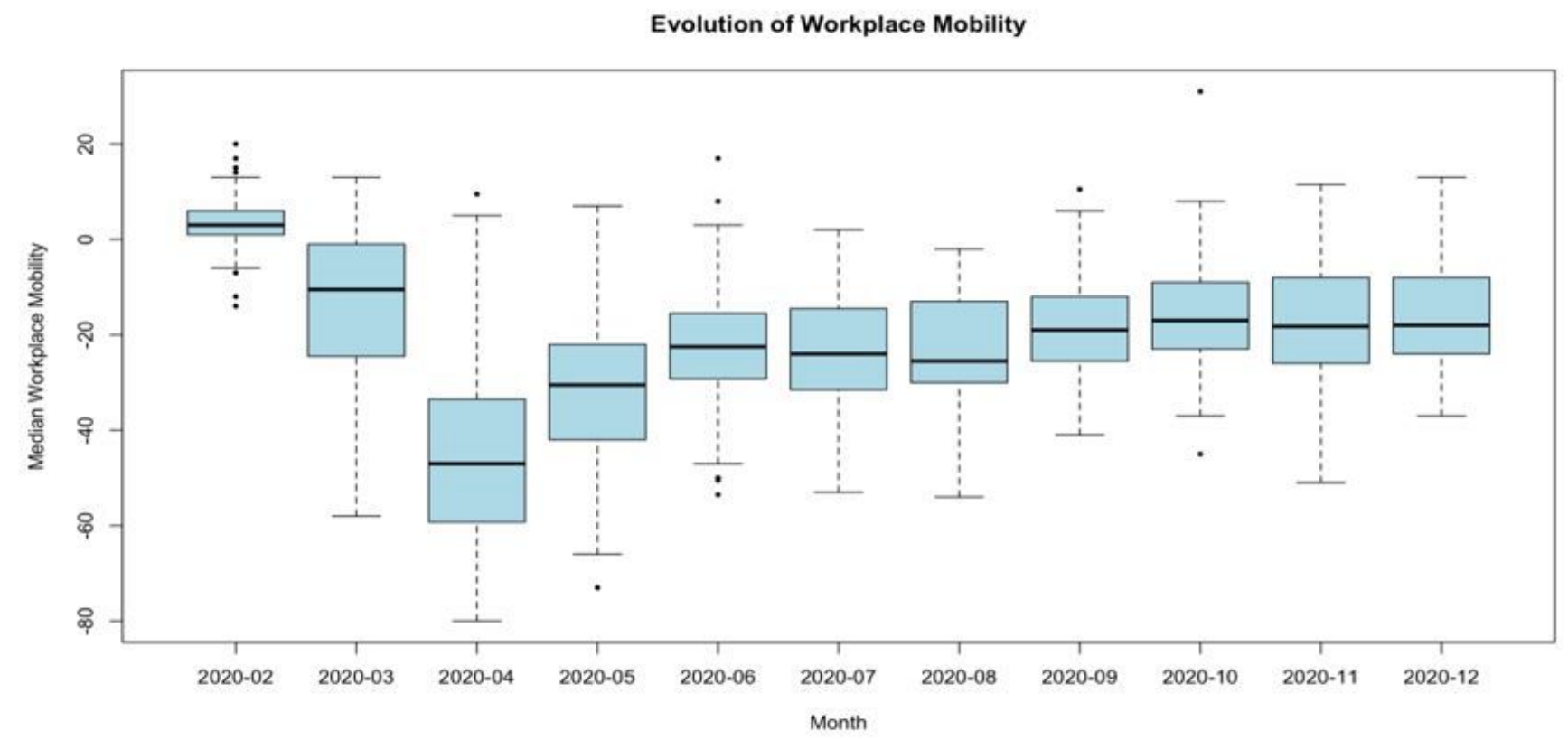

Figure 1

Evolution of workplace mobility. Boxplot of the average workplace mobility observed for each country in that month ( $n=116$ countries). The solid line in the box indicates the median, with boxes at the interquartile range. Whiskers are either at $1.5 *$ (interquartile range) outside the box, or at the extreme value. 
Evolution of Stay at Home Requirements

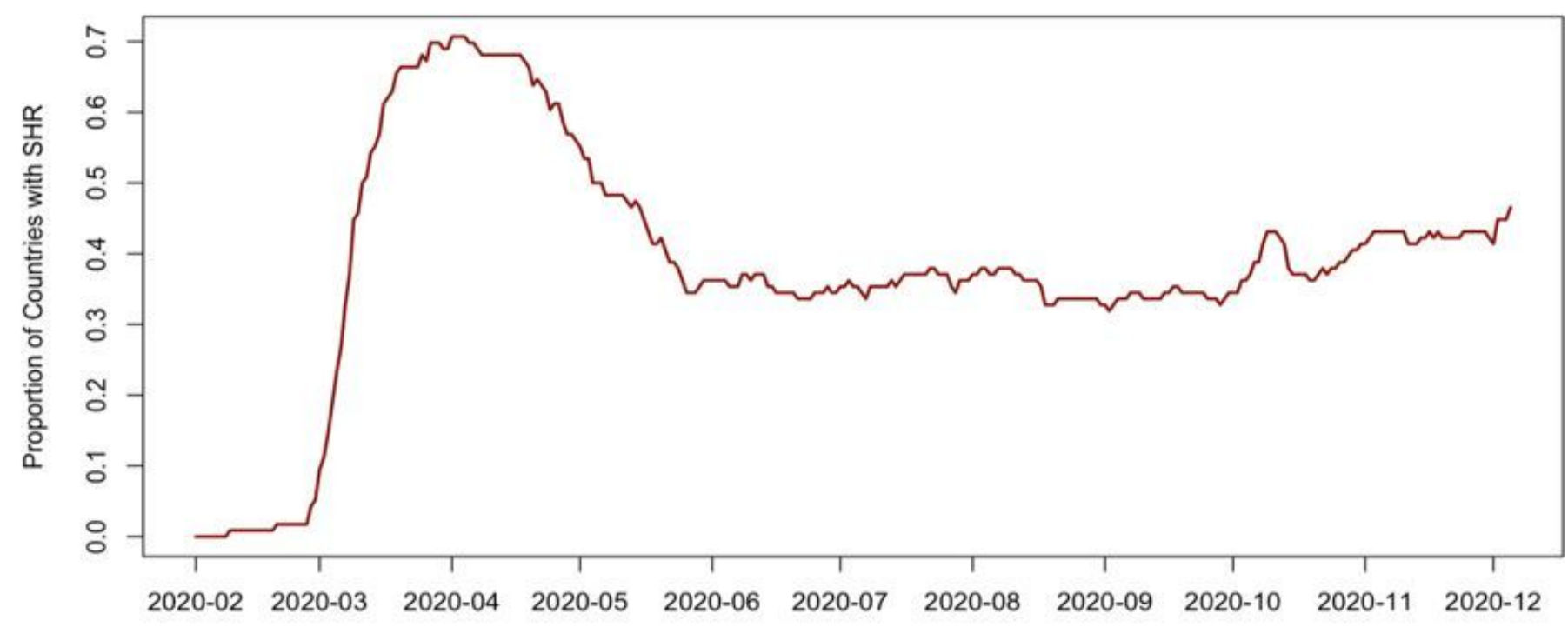

Figure 2

The proportion of countries with a lockdown. The daily fraction of countries that have an active stay-athome order for its general population ( $n=116$ countries).

\section{Evolution of Workplace Mobility During Lockdowns}

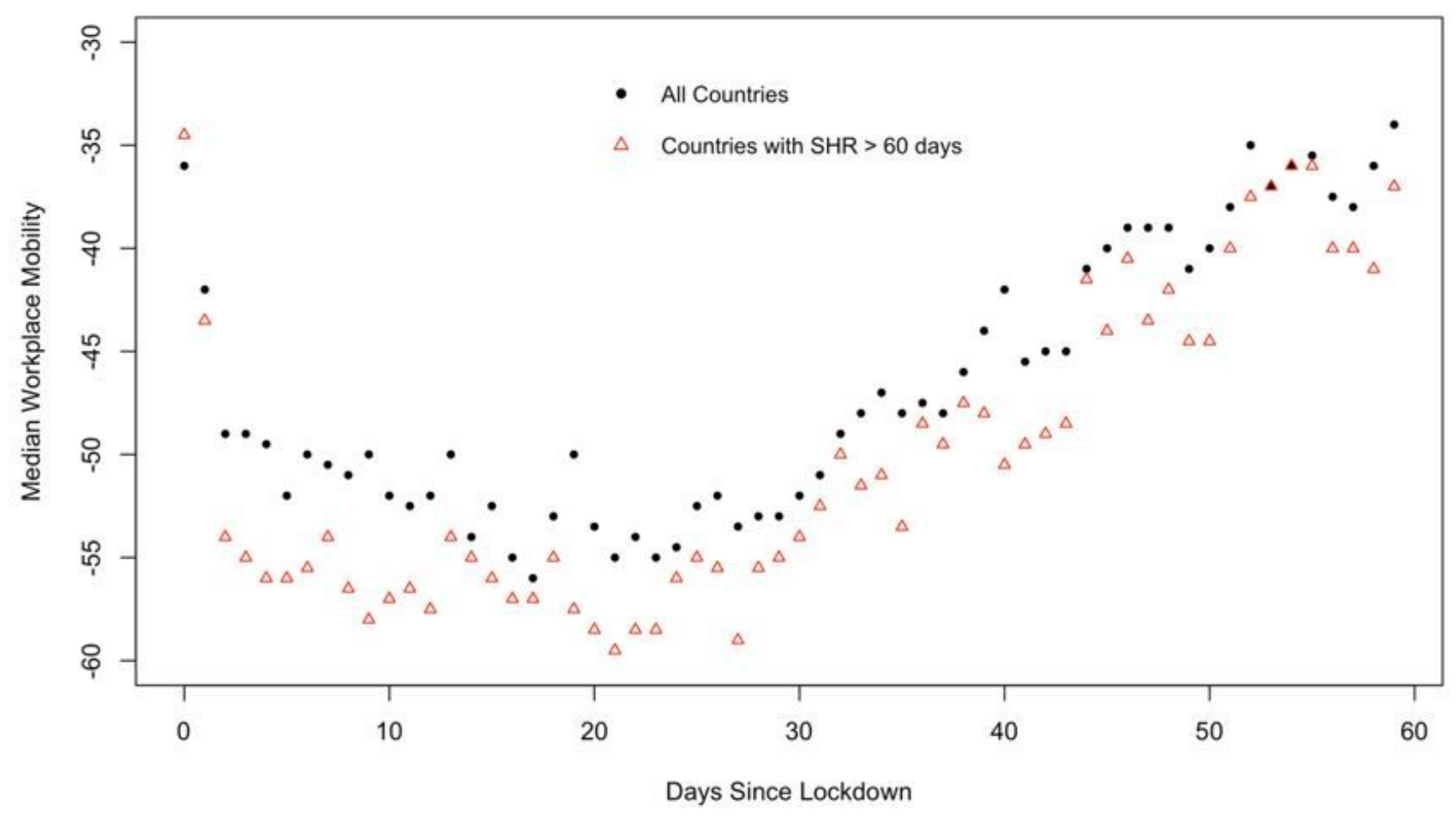




\section{Figure 3}

Evolution of workplace mobility during lockdowns. For each country ( $n=93$, solid dots), we identify the date when the first lockdown requirement was imposed over its general population. During the lockdown period, for each day since the start of the lockdown we determine the median workplace mobility across countries for that day. As a robustness test, this analysis is repeated for countries where the first lockdown lasted for more than 60 days ( $n=50$, hollow triangles).

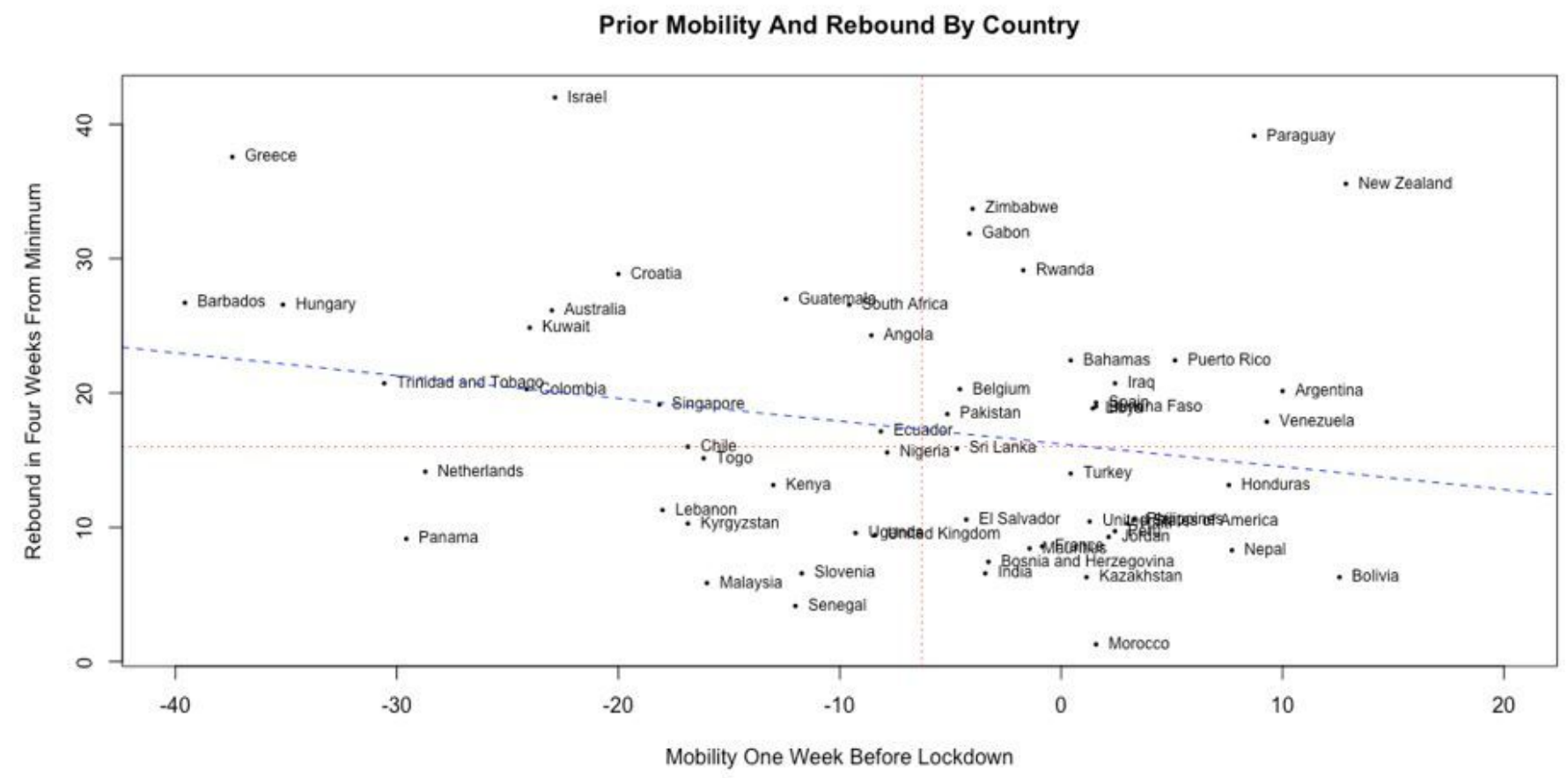

\section{Figure 4}

Prior mobility and rebound across $n=59$ countries. The horizontal axis represents the average mobility level one week before the start of the lockdown. The vertical axis represents the rebound in mobility in four weeks starting from the day when minimum mobility levels were achieved during the lockdown. 


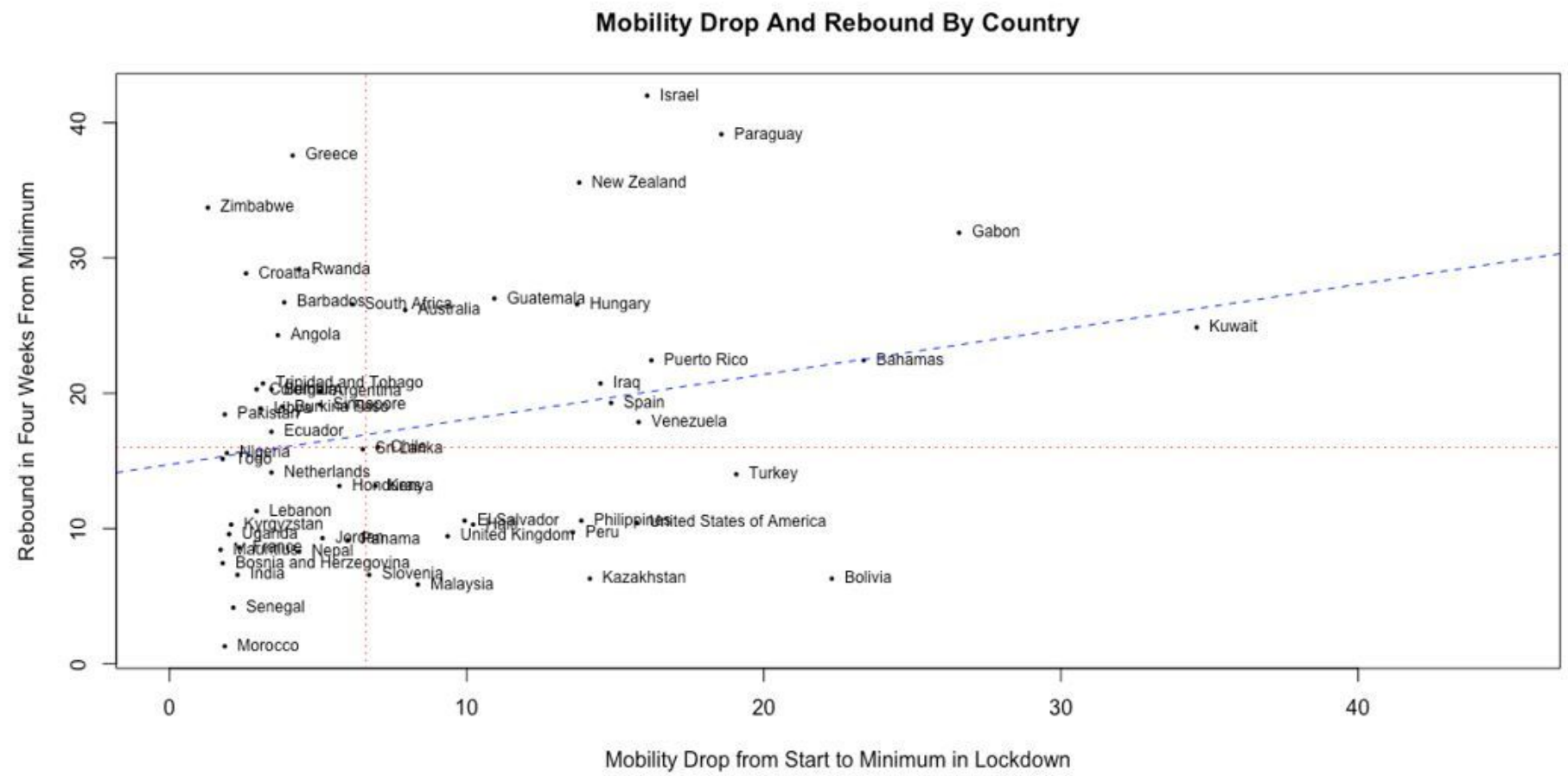

Figure 5

Mobility drop and rebound across $n=59$ countries. The horizontal axis represents the mobility drop from the start of the lockdown until reaching the minimum mobility level during the lockdown. The vertical axis represents the rebound in mobility in four weeks from the day when minimum mobility levels were achieved during the lockdown. 